\title{
Searches for neutrinos from Gamma-ray bursts with ANTARES
}

\author{
Julia Schmid ${ }^{a}$, Damien Turpin ${ }^{* b}$, on the behalf of the ANTARES collaboration \\ ${ }^{a}$ Friedrich-Alexander-Universität Erlangen-Nürnberg, Erlangen Centre for Astroparticle \\ Physics, Erwin-Rommel-Str. 1, 91058 Erlangen, Germany ${ }^{\dagger}$ \\ ${ }^{b}$ Aix Marseille Université, CNRS/IN2P3, CPPM UMR 7346, 13288, Marseille, France \\ E-mail: julia.schmidefau.de, damien.turpin@irap.omp.eu
}

\begin{abstract}
ANTARES is the largest high-energy neutrino telescope in the Northern Hemisphere. Its main scientific purpose is the search for astrophysical muon neutrinos that are detected via their chargedcurrent interaction in Earth and the subsequent Cherenkov emission of the secondary muon in the water of the Mediterranean Sea. Gamma-ray bursts are among the most promising candidates for the experiment as they are thought to accelerate not only electrons - leading to the observed gamma rays - but also protons, which would yield the emission of EeV neutrinos. Compelling evidence of a high-energy cosmic neutrino signal correlated with any astrophysical source would, for the first time, prove the acceleration of hadrons beyond any doubt, a hypothesis that cannot unambiguously be put to the test by pure electromagnetic observation. However, to explain the origin of cosmic rays at ultra-high energies, it is absolutely crucial to identify those processes in the universe that are capable of accelerating baryons to such energies. The recent searches for muon neutrinos from gamma-ray bursts using data of the ANTARES telescope will be presented, including constrains that can be put on individual model parameters and a scan for possibly timeshifted neutrino signals.
\end{abstract}

The 34th International Cosmic Ray Conference,

30 July- 6 August, 2015

The Hague, The Netherlands

\footnotetext{
* Speaker.

†now at Laboratoire AIM, CEA-IRFU/CNRS/Université Paris Diderot, Service d'Astrophysique, CEA Saclay, 91191 Gif sur Yvette, France
} 


\section{Introduction}

The detection of a high-energy neutrino signal from Gamma-ray bursts (GRB) would unambiguously probe them as powerful accelerators of hadrons. In the prevailing fireball model as proposed for example by Mészáros and Rees [1], the observed electromagnetic radiation is explained by synchrotron radiation and subsequent inverse Compton scattering of relativistic shockaccelerated electrons. Waxmann \& Bahcall [2] first suggested that there could be a significant baryonic loading in GRB jets (mainly protons). If these protons are sufficiently accelerated, they interact with the ambient photon field and produce neutral and charged pions. Subsequent decay of the latter would yield a high-energy neutrino signal associated with the electromagnetic GRB signal. Neutrino astronomy can therefore provide an unique tool to probe the nature and dynamics of GRB's jets and could also serve to explain the origin of the cosmic-ray flux at ultra-high energies.

The underwater neutrino telescope ANTARES [3] is primarily designed to detect cosmic muon-neutrinos in the $\mathrm{TeV}-\mathrm{PeV}$ range below the local horizon. In these proceedings, we present recent searches for muon-neutrino emission from GRBs using the ANTARES data.

\section{The NeuCosmA model}

Neutrino predictions are based on the photohadronic interactions between the accelerated protons and the ambient photon field. The first commonly used models of Waxmann \& Bahcall [2] and Guetta [4] have already been ruled out by the IceCube collaboration [5]. The NeuCosmA model [6],[7] is one of the up-to-date models that takes into account the full proton-photon cross section, including $\Delta^{+}$resonances, multiple pion and Kaon production which contributes to the highest energy part of the neutrino spectrum. The predicted neutrino spectrum depends on a set of 10 parameters describing the $\gamma$-ray prompt spectrum and the dynamics of the GRB jet: $F_{V}=f\left(z, \alpha_{\gamma}, \beta_{\gamma}, E_{p}, F_{\gamma}, \frac{\varepsilon_{e}}{\varepsilon_{B}}, \Gamma, f_{p}, t_{v a r}, T_{90}\right)^{1}$ where $z$ is the cosmological redshift of the burst,$\alpha_{\gamma}$ and $\beta_{\gamma}$ are the low and high energy spectral indexes of the $\gamma$-ray spectrum, $E_{p}$ is the peak energy of the observed $v F_{v} \gamma$-ray spectrum, $F_{\gamma}$ is the $\gamma$-ray fluence, $\varepsilon_{e}$ and $\varepsilon_{B}$ are the fraction of the internal jet's energy in electrons and in the magnetic field, $\Gamma$ is the bulk Lorentz factor, $f_{p}$ is the baryonic loading, $t_{v a r}$ is the minimum variability timescale of the $\gamma$-ray prompt emission and $T_{90}$ gives the burst duration.

\section{Searches for neutrinos from GRB110918A and GRB130427A}

The search methodology follows the one developed and applied in [8] on the ANTARES data from end of 2007 to end of 2011. It relies on the optimisation of the model discovery potential (MDP) applying per-GRB selection cuts on the track reconstruction quality parameter. In doing so, the likelihood ratio of signal (derived from Monte Carlo simulations) to background (based on data) is maximised. In a sample of 296 long GRBs from 2007 to 2011, no neutrino events were detected within the accumulated coincident search duration of 6.6 hours, where 0.06 neutrino events where predicted from the NeuCosmA model on a background of 0.05. An upper limit at $90 \%$ confidence

\footnotetext{
${ }^{1}$ The commonly-used default values for these parameters are $: z^{\text {def }}=2.15, \alpha^{\text {def }}=-1, \beta^{\text {def }}=-2, E_{p}^{\text {def }}=200 \mathrm{keV}$, $F_{\gamma}^{\mathrm{def}}=-10^{-5} \mathrm{ergcm}^{-2}, \varepsilon_{e}^{\mathrm{def}}=0.1, \varepsilon_{B}^{\mathrm{def}}=0.1, \Gamma^{\mathrm{def}}=316, f_{p}^{\mathrm{def}}=10, t_{\text {var }}^{\mathrm{def}}=0.01 \mathrm{~s}, T_{90}^{\mathrm{def}}=30 \mathrm{~s}$ (our choice here)
} 


\begin{tabular}{c|c|c|c}
\hline \hline Scanned parameter & $\mu_{s}^{\min }$ & $\mu_{s}^{\max }$ & $\delta \mu_{s}$ \\
\hline$\Gamma$ & $5.6 \times 10^{-8}(\Gamma=900)$ & $0.12(\Gamma=60)$ & $2.1 \times 10^{6}$ \\
$f_{p}$ & $4.9 \times 10^{-6}\left(f_{p}=0.5\right)$ & $9.7 \times 10^{-4}\left(f_{p}=200\right)$ & 198.0 \\
$\frac{\varepsilon_{e}}{\varepsilon_{B}}$ & $3.3 \times 10^{-5}\left(\frac{\varepsilon_{e}}{\varepsilon_{B}}=0.01\right)$ & $5.5 \times 10^{-5}\left(\frac{\varepsilon_{e}}{\varepsilon_{B}}=100\right)$ & 1.7 \\
\hline
\end{tabular}

Table 1: Results of the parameter scans. GRB standard values were used for the fixed parameters, see section 2. The minimum $\mu_{s}^{\min }$ and maximum $\mu_{s}^{\max }$ numbers of neutrinos obtained during the different scans are indicated with the associated parameter value. $\delta \mu_{s}$ measures the absolute variation of the expected number of neutrinos inside the parameter space of $\Gamma, f_{p}$ and $\frac{\varepsilon_{e}}{\varepsilon_{B}}$ according to the NeuCosmA predictions for long GRBs.

has hence been derived [8]. The total predicted neutrino flux was mainly dominated by the very energetic and relatively close burst GRB110918A. This means that the detection of a neutrino signal from an individual GRB is very unlikely except for particular energetic bursts as GRB110918A. The very nearby burst GRB130427A was also in the ANTARES field of view and was considered as a promising candidate for a neutrino detection. Thus a specific search for a neutrino signal was performed, where the spectral and temporal properties were collected from [9]. From the NeuCosmA model, $6.2 \times 10^{-3}$ events were expected, yielding a $3 \sigma \mathrm{MDP}$ of $0.86 \%$. Equivalently for GRB110918A, a $3 \sigma$ MDP of 3.25\% has been derived using NeuCosmA. No coincident neutrino signal has been observed, consequently derived upper limits after non-detection are shown in Fig. 1.

\section{ANTARES constraints on the physics of GRB110918A and GRB130427A}

One should note that the NeuCosmA predictions were determined with standard values for the non-measured physical parameters, i.e. $\Gamma=316, f_{p}=10$ and $\frac{\varepsilon_{e}}{\varepsilon_{B}}=1$ and could strongly bias the final result. In order to evaluate how the unknown parameters impact the NeuCosmA expectations, the entire parameter space was scanned within expectations for long GRBs. For each parameter set, standard values were assumed for the fixed parameters and the expected numbers of neutrinos $\mu_{s}^{i}$ were derived accounting for the time-average ANTARES effective area from 2007 to 2011. The influence of each parameter on the neutrino expectations is given by the ratio between the maximum and the minimum number of predicted neutrinos: $\delta \mu_{s}=\max \left(\mu_{s}^{i}\right) / \min \left(\mu_{s}^{i}\right)$. As shown in Table 1, the bulk Lorentz factor $\Gamma$ and, to a smaller extent, the baryonic loading $f_{p}$, crucially influence the neutrino predictions. For instance, a GRB with a high Lorentz factor $(\Gamma \sim 900)$ would exhibit $\sim 10^{6}$ less neutrinos than the same GRB with a low $\Gamma \sim 60$ according to NeuCosmA. On the other hand, the ratio $\frac{\varepsilon_{e}}{\varepsilon_{B}}$ has only minor influence in the neutrino expectations. Hence it is possible to constrain regions in the $\Gamma$ and $f_{p}$ parameter space by excluding models that would predict a detectable signal at the $90 \%$ confidence level $\left(\mu_{s} \geq 2.3\right)$.

$10000 \mathrm{NeuCosmA}$ spectra were generated for each burst in order to cover the whole range of $\Gamma \in[10 ; 900]$ and $f_{p} \in[0.5 ; 200]\left(\frac{\varepsilon_{e}}{\varepsilon_{B}}\right.$ was fixed at its standard value). For each simulated spectrum, the expected number of neutrinos $\mu_{s}$ was calculated by taking into account the ANTARES effective area for GRB110918A and GRB130427A. The ANTARES constraints on $\Gamma$ and $f_{p}$ for these two 


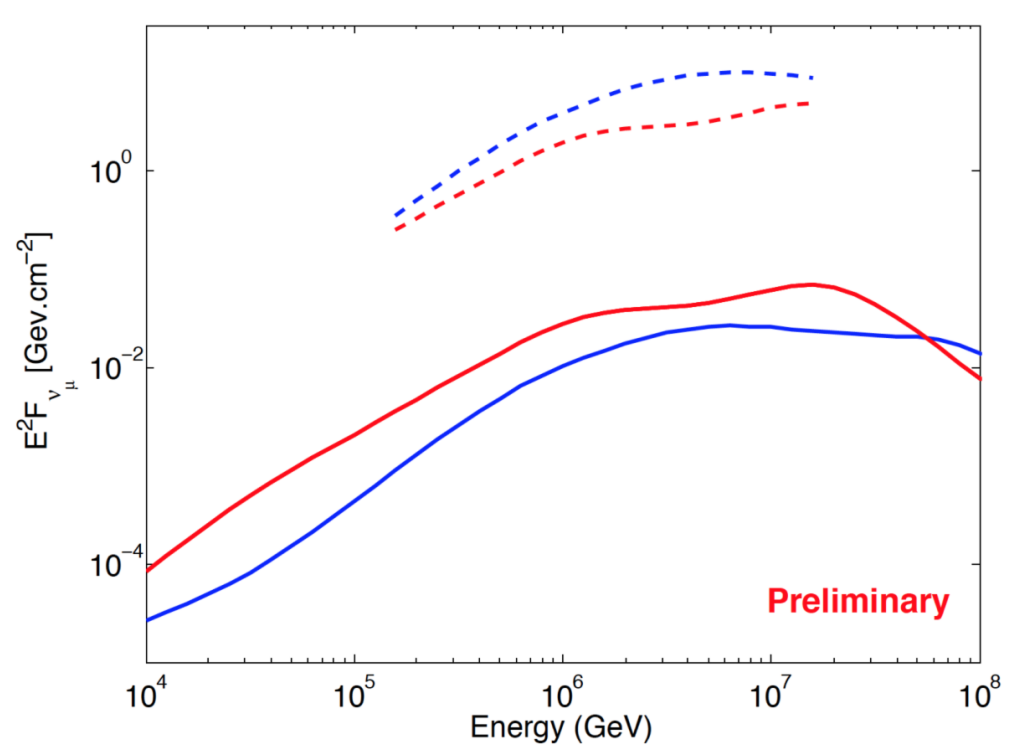

Figure 1: NeuCosmA spectra $\left(v_{\mu}+\bar{v}_{\mu}\right)$ for GRB110918A (red) and GRB130427A (blue). The dashed lines indicate the derived limits on the coincident neutrino emission with GRB110918A (red) and GRB130427A (blue) in the energy range where $90 \%$ of the signal is expected to be detected.
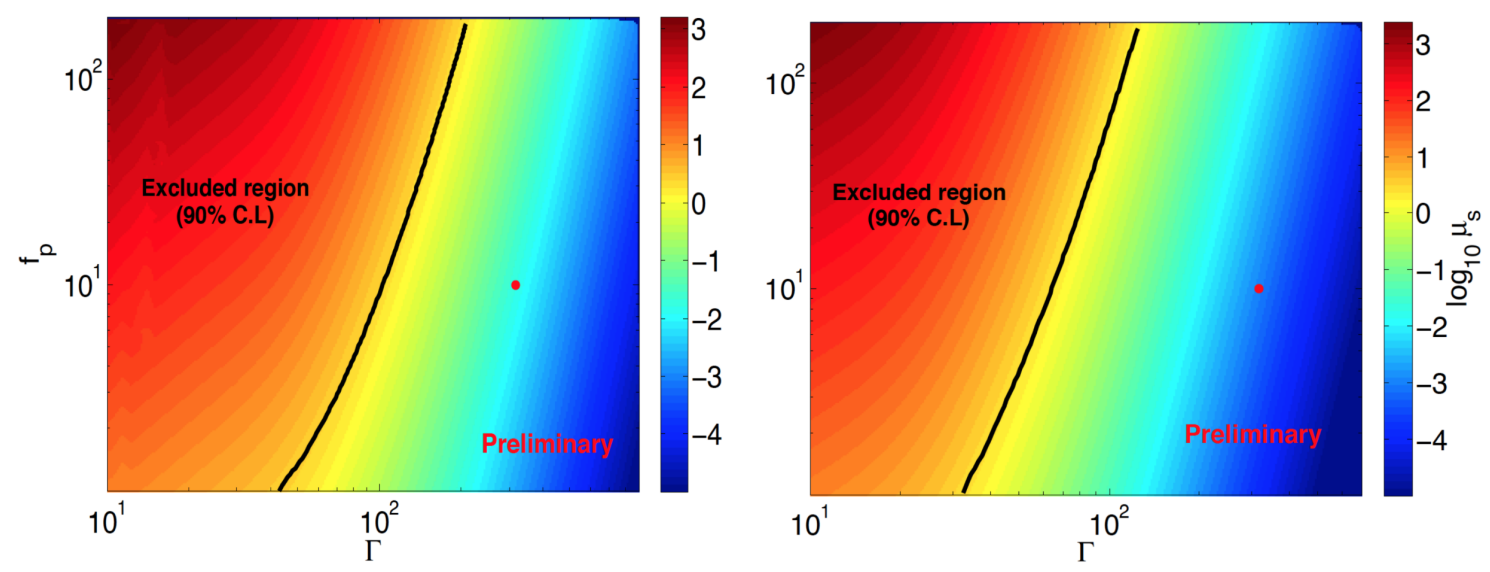

Figure 2: Expected number of neutrinos (color coded) as function of $\Gamma$ and $f_{p}$ for GRB110918A (lefthand panel) and GRB130427A (right). The black line indicates the region excluded by ANTARES at $90 \%$ confidence level $\left(\mu_{s} \geq 2.3\right)$, the red dots shows the standard values of $\Gamma$ and $f_{p}$ for long GRBs.

burst do not strongly challenge the standard predictions of the internal shocks model as shown in Fig.2.

\section{Search for time-shifted neutrino emission from gamma-ray bursts}

Up to now no neutrino signal could be identified above the background in the data from any neutrino detector during the prompt emission phases, and the first optimistic analytical models 
have already been challenged by IceCube [5]. Even though the search for a signal of neutrinos coincident with the emission of high-energy photons is the most generic ansatz, there are many models that predict time-shifted neutrino signals, such as neutrino precursors [10] or afterglows [11], or different Lorentz Invariance Violation (LIV) effects for photons and neutrinos on their way to Earth [12]. Thanks to their cosmic distances and transient nature, gamma-ray bursts provide unique test environments to study and verify such effects. A novel model-independent technique was developed to distinguish a time-shifted neutrino signal from the expected background, which allows even faint signals to be detected using a large sample of GRBs. The search relies on stacked time profiles of neutrinos spatially coincident with GRBs in a wide time window which would enable to detect a systematic shift of neutrinos (from emission or propagation) with respect to the electromagnetic emission. Any neutrino emission associated with the GRBs, even if faint, would give rise to a cumulative effect in these stacked profiles, which can then be identified by its discrepancy from randomised data.

The neutrino candidate sample for the search for neutrino point-sources [13] provides naturally suited data for this approach. The stringent quality cuts guarantee low muon background contamination and excellent angular resolution. This data sample consists of 5516 neutrino candidate events from March 2007 to the end of 2012. A suitable gamma-ray-burst sample was consolidated similarly to the one used in [8].

Several observables sensitive to different potential origins of such a shift were considered. The simplest one is a delayed detection time $\tau=t_{v}-t_{\mathrm{GRB}}$ of neutrinos with respect to the detected gamma-rays. Another one, sensitive to potential shifted emission times, is corrected for the redshift $\tau_{z}=\frac{\tau}{1+z}$. Potential LIV effects are, on first order, supposed to be linearly dependent on energy[12], so we define the measure $\tau_{\mathrm{LIV}}=\frac{\tau}{E_{\text {est. }} \cdot D(z)}$, with the estimated neutrino energy $E_{\text {est }}$ and the distance of the GRB $D(z)$ as defined in [12]. From the stacked histograms of these observables, we construct a test statistic [14]:

$$
\psi=-10\left[\log _{10} n !+\sum_{k=1}^{m} n_{k} \log _{10} p_{k}-\log _{10} n_{k} !\right],
$$

with the total number of events $n$ being distributed in the $k \in[1 \ldots m]$ bins with probability $p_{k}$.

An optimal choice of the search cone size $\delta_{\max }$ naturally depends on the gamma-ray burst's position accuracy and the neutrino pointing uncertainty of the detector. We chose a per-GRB coincidence cone size by optimising the ratio of signal to square root of noise of a two-dimensional gaussian signal on flat background[15]:

$$
\delta_{\text {cut }}=1.58 \cdot \max \left(\sigma_{v}, \Delta_{\text {err }, \mathrm{GRB}}, \theta_{\mathrm{lim}}\right) .
$$

where $\sigma_{v}$ is the neutrino sample median resolution and $\Delta_{\text {err, GRB }}$ the size of the GRB error box. The cone size was limited by $\theta_{\text {lim }}$ such that no single GRB contributes more than an order of magnitude more background than another.

The size of the probed time window $\tau_{\max }$ should be defined as the largest shift predicted by any of the models. The largest arrival time delays between neutrinos and gamma-rays could be introduced by LIV effects. Considering the most recent limit on the potential LIV energy scale [16] and the highest measured GRBs' redshift so far $(z \sim 9)$, we limited the maximum considered time shift to 40 days. These choices reduced the initial sample to 563 GRBs occurring below the local ANTARES horizon (and 150 with measured redshift). 


\subsection{Sensitivity and results}

To investigate the performance of the proposed technique to identify hypothetical neutrinos from GRBs, a test signal was mimicked by associating neutrino candidates artificially with part of the GRBs at an (hypothetical) intrinsic time shift of five days. That is, taking into account the cosmological redshift $z$, a simulated signal delayed by $t_{v}=t_{\mathrm{GRB}}+5 \mathrm{~d} \cdot(1+z)$. The sensitivity, defined as the $90 \%$ confidence-level upper limit that can be placed on the number of GRBs that produced an associated neutrino signal in the ANTARES data when observing the median background, is $m\left(f_{\text {all }}^{90 \% \mathrm{CL}}\right)=0.6 \%$, as shown in Fig. 3. Considering only the sub-sample of bursts with determined redshift, the method is even sensitive to a signal in only $1.1 \%$ of the bursts, which corresponds to $0.3 \%$ of the entire sample.

When applying the search to actual ANTARES data, no events were found in coincidence with the GRBs where 4.4 were expected from purely randomised data $(0.7$ for GRBs with measured redshift) which is an under-fluctuation of $1.2 \%$ probability $(51.4 \%)$. This low probability prevents us from putting a limit at the standard $90 \%$ confidence level. However, we can exclude any signal in more than $0.06 \%$ of the bursts with a $99 \%$ confidence.

We have performed the same search with the IceCube public IC40 point source search sample [17]. It consists of 12877 neutrino collected between April 2008 and May 2009 that have been searched for associations with $40 \mathrm{GRBs}$ (12 with redshift measurement). In total, 42 neutrinos (8) are found in coincidence where 35 (4) were expected from randomised data. These slight excesses with $p$-value of $13.5 \%(5.1 \%)$ are still compatible with the background expectations. This result derived on a complementary GRB sample as well as numerous cross-checks testing different coincidence selections confirms the fact that the observed underfluctation in the ANTARES data sample is not introduced by systematic effects of the method or the software, but is indeed inherent in the considered data sample.

\section{Discussion and conclusion}

Following the stacked search for neutrinos during the prompt emission of GRBs in the data of ANTARES between late 2007 and 2011, the individual analysis of the exceptionally nearby GRB130427A has also not revealed any significant signal excess. Consequently, upper limits on the neutrino fluxes have been derived. Given the ANTARES sensitivity, only the most extreme physical parameters can be excluded. Nevertheless, the non-observation of a neutrino signal from these bursts by the ANTARES and IceCube collaborations is a strong indication that the bulk Lorentz factor of the most energetic GRBs could be very high. It has been shown that the expected NeuCosmA neutrino signal for a GRB with a high $\Gamma$ value can be up to six orders of magnitude lower than for a GRB with low $\Gamma$. Thus, the ideal GRB to produce high-energy neutrino emission would be a very luminous burst with a moderate Lorentz factor. Note, however, that these results are interpreted in the framework of internal shock models of GRBs. Other models predict highenergy neutrino emission with more or less efficiency at different places in the jet. For instance, the photospheric model [18], [19] predicts higher neutrino flux at lower energy and will be included in future investigations. 

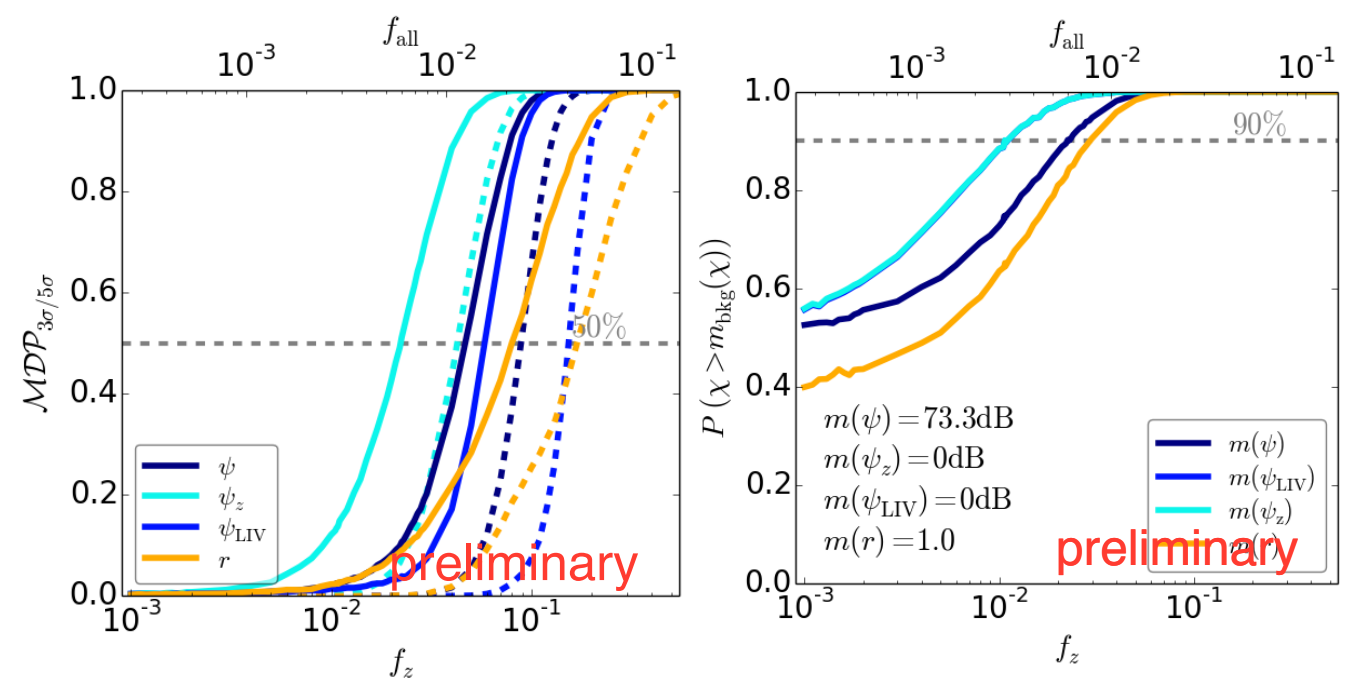

Figure 3: Efficiencies or detection probability $P$ at $3 \sigma$ (solid) and $5 \sigma$ (dashed lines) for the test statistics and the ratio $r$ of events before and after the GRB alert as a function of the mean fraction $f$ of GRBs with one associated signal neutrino at $t_{v}=t_{G R B}+5 \mathrm{~d} \cdot(1+z)$ (left). The fraction $f_{z}$ denotes the fraction of GRBs with one associated signal neutrino in the ANTARES data with determined redshift $z$, whereas $f_{\text {all }}$ gives the fraction of the whole GRB sample. On the right: Probabilities $P$ to measure values of the test statistics above the median value from the background-only realizations. The sensitivity is given by the signal fraction $f$ where the curves reach $90 \%$ probability (gray dashed line). Probabilities were derived using the ANTARES data from 2007-2012.

We also performed a model-independent search for time-shifted neutrinos with respect to the prompt emission of GRBs and could not identify any excess. This enables us to put a limit on the average fraction of GRBs that might produce a detectable neutrino signal in the ANTARES data, even if shifted in time, to about $1 \%$. The future larger neutrino telescope KM3NeT with significantly increased sensitivity of up to a factor of $\sim 50$ will be able to challenge the neutrino predictions in the framework of the GRB fireball model. In the meanwhile, the collection of more ANTARES data is still ongoing and will help to improve the neutrino flux limit, providing at the same time a continuous monitoring of GRB neutrino emission in the Southern Hemisphere.

\section{Acknowledgements}

We would like to thank Mauricio Bustamante for helpful discussions and making it possible to use the NeuCosmA model. J. Schmid would like to thank the Studienstiftung des Deutschen Volkes for their financial support. D. Turpin would also gratefully acknowledge financial support from the OCEVU LabEx, France.

\section{References}

[1] Mészáros, P. \& Rees, M. J. 1993, The Astrophysical Journal, 405, 278-284

[2] Waxman, E. \& Bahcall, J. 1997b, Physical Review Letters, 78, 2292

[3] Ageron, M., Aguilar, J. A., Al Samarai, I., et al. 2011, Nucl. Instr. Meth. A, 656, 11 
[4] Guetta, D., Spada, M., \& Waxman, E. 2001, The Astrophysical Journal, 559, 101

[5] Abbasi, R., Abdou, Y., Abu-Zayyad, T., et al. 2012, Nature, 484, 351

[6] Hümmer, S., Rüger, M., Spanier, F., \& Winter, W. 2010, The Astrophysical Journal, 721, 630

[7] Hümmer, S., Baerwald, P., \& Winter, W. 2012, Phys. Rev. Lett., 108, 231101

[8] Adrián-Martínez S. et al., A\&A, 559 (2013) A9

[9] von Kienlin, A. 2013, GRB Coordinates Network, 14473

[10] Razzaque S. et al., 2003, Phys. Rev. D 68, 083001 Amelino-Camelia, G., Guetta, D., \& Piran, T. 2013, ArXiv e-prints [arXiv:1303.1826]

[11] Waxman E., 2000, ApJS 127, 519

[12] Amelino-Camelia et al. 2013, ArXiv e-prints [arXiv:1303.1826]

[13] Adrián-Martínez S. et al., 2014, Astrophys. J., Lett. 786, L5

[14] van Eijndhoven N., 2008, Astroparticle Physics 28, 540

[15] Alexandreas D.E. et al., 1993, Nucl. Instr. Meth. A 328, 570

[16] Vasileiou V. et al. 2013, Phys. Rev. D 87, 122001

[17] Abbasi, R. et al. 2011, ApJ., 732, 18

[18] Zhang, B. \& Kumar, P. 2013, Physical Review Letters, 110, 12

[19] Gao, S., Asano, K. \& Mészáros, P., 2012, JCAP, 11, 058 